\title{
The effect of supplementation with a calcium rich marine-derived multi-mineral supplement alone or in combination with short-chain fructo-oligosaccharide on body composition in postmenopausal women
}

\author{
B. E. Cronin, P. J. Allsopp, M. M. Slevin, P. J. Magee, M. B. E. Livingstone, J. J. Strain \\ and E. M. McSorley \\ Northern Ireland Centre for Food and Health, University of Ulster, Coleraine, BT52 1SA
}

This abstract was awarded the student prize for best original Poster Communication.

Calcium (Ca) supplements are renowned for their beneficial effect on bone health and are taken by postmenopausal women for the prevention of osteoporosis. A high Ca intake, particularly from dairy foods is associated with beneficial effects on body composition ${ }^{(1)}$ and weight status ${ }^{(2)}$. Ca supplements may impact favourably on the two components of body mass: fat mass (FM) and fat free mass (FFM); the evidence remains controversial. Short-chain fructooligosaccharides (scFOS) have been shown in vivo to increase Ca absorption $^{(3)}$. The aim of this study was to investigate the effects of a Ca rich marine-derived multi-mineral supplement administered alone or in combination with scFOS on body composition in postmenopausal women.

A total of 300 postmenopausal women (mean BMI $27.4($ SD 4.8$) \mathrm{kg} / \mathrm{m}^{2}$ ) aged 47-75 years were recruited to take part in a two year double-blind placebo controlled trial. Participants were randomly assigned to daily supplements of $800 \mathrm{mg}$ of Ca (2.4 g Aquamin ${ }^{\circledR}$ ), $800 \mathrm{mg}$ of Ca with $3 \mathrm{~g}$ of scFOSs (3.2 $\mathrm{g}$ Nutraflora $^{\circledR}$ ) (CaFOS) or maltodextrin (MD). Body mass index (BMI), waist circumference, FM, FFM and trunk fat (determined by dual energy x-ray absorptiometry) were measured at baseline and 24 months. Intention to treat analysis using ANCOVA (with baseline measures as covariates) was conducted to assess treatment effects between groups, controlling for age, baseline $\mathrm{Ca}$ intake (mean intake $868 \mathrm{mg} \mathrm{Ca}$ /day) alcohol use and physical activity.

There was no treatment effect for each of the body composition parameters assessed; BMI, waist circumference, FM, FFM and trunk fat. Supplementation with Aquamin, a Ca rich marine-derived multi-mineral supplement alone or in combination with scFOS has no effect on body composition in postmenopausal women over a two year period.

This work was funded by Marigot Ltd (Cork, Ireland), Ingredion Inc. (Westchester, IL) and a PhD studentship received from the Department for Employment and Learning.

1. Zhu W, Cai D, Wang Y et al. (2013) Nutr $J$ 12, 1-8.

2. Davies K, Heaney R, Recker R et al. (2000) J Clin Endocrinol Metab 85, 4635-8.

3. Morohashi T, Sano T, Ohta A et al. (1998) J Nutr 128, 1815-8. 